4. Reich K, Burden AD, Eaton JN, Hawkins NS. Efficacy of biologics in the treatment of moderate to severe psoriasis: a network meta-analysis of randomized controlled trials. Br J Dermatol 2012; 166: 179-88.

5. Talamonti $M$, Botti $E$, Galluzzo $M$, et al. Pharmacogenetics of psoriasis: HLA-Cw6 but not LCE3B/3C deletion nor TNFAIP3 polymorphism predisposes to clinical response to interleukin 12/23 blocker ustekinumab. Br J Dermatol 2013; 169: 458-63.

6. Leonardi $C L$, Kimball $A B$, Papp $K A$, et al. Efficacy and safety of ustekinumab, a human interleukin-12/23 monoclonal antibody, in patients with psoriasis: 76-week results from a randomised, double-blind, placebo-controlled trial (PHOENIX 1). Lancet 2008; 371: 1665-74

7. Talamonti M, Galluzzo M, Chimenti S, Costanzo A. HLA-C 06 and response to ustekinumab in Caucasian patients with psoriasis: outcome and long-term follow-up. J Am Acad Dermatol 2016;74: 374-5.

8. Chiu HY, Wang TS, Chan CC, Cheng YP, Lin SJ, Tsai TF. Human leucocyte antigen-Cw6 as a predictor for clinical response to ustekinumab, an interleukin-12/23 blocker, in Chinese patients with psoriasis: a retrospective analysis. Br J Dermatol 2014; 171: 1181-8.

9. Li K, Huang CC, Randazzo $B$, et al. HLA-C*06:02 allele and response to IL-12/23 inhibition: results from the ustekinumab Phase 3 psoriasis program. J Invest Dermatol 2016; 136: 2364-71.

10. Talamonti $M$, Galluzzo $M$, van den Reek JM, et al. Role of HLA- $C * 06$ in clinical response to ustekinumab: evidence from reallife in a large cohort of European patients. Br J Dermatol 2017; 177: 489-96.

doi:10.1684/ejd.2017.3071

\section{Non-melanoma skin cancers in African American solid organ transplant recip- ients: regional bias or a real need for surveillance?}

The rate of solid organ transplantation in non-white patients continues to increase. Our understanding of the epidemiology and risk of non-melanoma skin cancer (NMSC) in this population remains limited. Currently available data on NMSC in solid organ-transplant recipients (SOTRs) is predominantly derived from Caucasian patients. NMSC remains the most common neoplasm in the post-transplant setting. The incidence of squamous cell carcinoma (SCC), basal cell carcinoma (BCC), and melanoma is increased 65-fold, 10-fold, and 3fold, respectively, in comparison with ethnically-matched control populations [1]. Previously published studies in patients with ethnic skin, however, have been limited and report variable data [2-5]. A recent retrospective study on NMSC among 259 non-white SOTRs reported a total of 19 NMSCs in 15 patients (5.8\%); this is in contrast with an overall low risk described in previous reports [6].

We conducted a retrospective analysis of self-identified non-Caucasian SOTRs referred to the Department of Dermatology at Johns Hopkins Hospital (JHH) between 1990 and 2007 with a minimum follow-up time of five years. Patients' data and characteristics are shown in table 1. A total of 4,090 solid organ transplants were performed at JHH during this time frame, of which 1,114 patients selfidentified as non-Caucasian. Among 1,114 patients, 180 $(16 \%)$ were evaluated in the Department of Dermatology and were included in the study.
Table 1. Patient data and characteristics.

\begin{tabular}{|ll|}
\hline Characteristic & Number (\%) \\
\hline Ethnicity, No. (\%) & \\
Total number of patients & $180(100)$ \\
African American & $160(88.9)$ \\
Asian & $14(7.8)$ \\
Arab & $3(1.7)$ \\
Hispanic & $2(1.1)$ \\
Pacific Islander & $1(0.6)$ \\
Sex & \\
Men & $85(47.2)$ \\
Women & $95(52.8)$ \\
Transplanted organ & \\
Kidney & $110(61.1)$ \\
Heart & $18(10.0)$ \\
Lung & $13(7.2)$ \\
Liver & $12(6.7)$ \\
Kidney-Pancreas & $7(3.9)$ \\
Multiorgan & $20(11.1)$ \\
NMSC & \\
SCC & 4 \\
in situ & 2 \\
Invasive & $2^{\mathrm{a}}$ \\
SCC sun-exposed area & $2^{\mathrm{b}}$ \\
BCC non-sun-exposed area & $2^{\mathrm{c}}$ \\
\hline
\end{tabular}

${ }^{a}$ in situ with focus of invasion ${ }^{b}$ cheek and arm $^{c}$ inguinal crease and labia

African Americans (AAs) represented the largest group of patients $(n=160 ; 89 \%)$. A total of six NMSCs were observed in four $(2.5 \%)$ of the AA cohort. These included two squamous cell carcinomas in situ (SCCIS), one SCCIS with focus of invasion, one invasive SCC, and two BCCs. Three $(3.9 \%)$ AA males $(n=77)$ and one $(1.2 \%)$ female $(n=83)$ developed NMSC. These patients had received renal $(n=2)$, heart $(n=1)$, and lung $(n=1)$ transplants. Two SCC developed in non-sun-exposed areas (inguinal region, labja majora).

The main limitation of our retrospective analysis is the small sample size. However, the predominance of SCC remains consistent with previous reports, which show SCC as the most common NMSC among AAs, affecting 3 per 100,000 patients [7]. Furthermore, we only analysed a small proportion of patients (16\%), who were examined in our dermatology clinic and had a histological diagnosis. Our study design represents the true referral bias based on chief complaint and perceived risk in this population. Appropriate dermatological evaluation of the entire cohort of patients with ethnic skin $(1,114)$ may have had a significant impact on our observed incidence and the types of cutaneous neoplasms encountered. Potential risk factors for NMSC, such as sun exposure, family history, and smoking, were not analysed, given the variable availability of data in the retrospective analysis. Lastly, the reported ethnicity was derived from the patients' history (self-report), which by itself introduces a bias.

Despite these limitations, our results support a higher risk of NMSC development in AA SOTRs than previously suspected in AAs. The observation of SCC in non-sun-exposed skin combined with patients' perception of their skin colour 
and lowered inherent risk of skin cancer development, with biased referral patterns, may all contribute to a delay in, or missed, diagnosis. Continuing to elucidate risk factors for NMSC development in the growing population of nonCaucasian transplant recipients is imperative as it will allow us to develop more directed screening resources, counselling, and educational tools.

Disclosure. Financial support: none. Conflict of interest: none.

${ }^{1}$ Division of Immunology, Allergy, Infectious Diseases, Department of Dermatology, Medical University of Vienna, Vienna,

Austria

${ }^{2}$ Department of Dermatology,

Johns Hopkins School of Medicine,

Baltimore, MD, USA

$<$ liliane.borik@meduniwien.ac.at>

1. Euvrard S, Kanitakis J, Claudy A. Skin cancers after organ transplantation. N Engl J Med 2003; 348: 1681-91.

2. Kasiske BL, Snyder JJ, Gilbertson DT, Wang C. Cancer after kidney transplantation in the United States. Am J Transplant 2004; 4: 905-13.

3. Gruber SA, Singh A, Mehta K, Morawski K, West MS, Doshi MD. Different patterns of cancer incidence among African American and Caucasian renal allograft recipients. Surgery 2010; 148: $661-5$.

4. Moosa MR. Racial and ethnic variations in incidence and pattern of malignancies after kidney transplantation. Medicine (Baltimore) 2005; 84: 12-22

5. Imao $T$, Ichimaru $N$, Takahara $S$, et al. Risk factors for malignancy in Japanese renal transplant recipients. Cancer 2007; 109: 2109-15.

6. Pritchett EN, Doyle A, Shaver CM, et al. Non-melanoma skin cancer in non-white organ transplant recipients. JAMA Dermatol 2016; 152: 1348-53

7. Gloster HM Jr., Neal K. Skin cancer in skin of color. J Am Acad Dermatol 2006; 55: 741-60.

doi:10.1684/ejd.2017.3059

\section{Efficacy of biologics in psoriasis patients under hemodialysis}

The biologics anti-tumour necrosis factor (TNF)- $\alpha$, anti-interleukin (IL)-12, and IL-23 are indicated for severeto-moderate plaque psoriasis in patients who fail to respond, or have contraindication, to systemic treatments or phototherapy. Chronic kidney disease (CKD) is a contraindication for many treatments. There are limited data about the use of biologics in CKD. No contraindication nor renal toxicity are reported, and the pharmacokinetic and impact of dialysis on their efficacy are unknown. The objective of this study was to investigate the efficacy of biologics in severe psoriasis patients under hemodialysis, as well as the impact of dialysis on plasmatic concentrations of these molecules.
In this retrospective multicentre study throughout France (RésoPso), we analysed the data of five patients with severe psoriasis treated with biologics who were receiving hemodialysis for CDK, without psoriatic arthritis. One patient was treated with ustekinumab, three with etanercept, and one with infliximab. The treatment was effective in five cases, even under hemodialysis, with PASI 75 or PASI 100. Measurement of serum concentrations of the molecules used were carried out by Theradiag (LISATRAKER box; ELISA method). For Patient 1, plasmatic ustekinumab concentration decreased gradually with repetition of dialysis sessions between two injections; the concentration was decreased two-fold at five weeks, and became undetectable at 10 weeks. Anti-ustekinumab antibodies were negative. For Patient 4, plasmatic etanercept concentrations appeared stable between two injections despite dialysis sessions. Anti-etanercept antibodies were negative. For these two molecules, one single dialysis session appeared to slightly increase plasmatic concentrations (figure 1).

There are limited data on the use of biologics in hemodialysis patients, especially for psoriasis. Pharmacokinetics are not well known, but no dose adjustment is necessary. Etanercept is an anti-TNF $\alpha$, the half-life of which is about 68 to 102 hours in patients with normal renal function. Plasmatic concentrations are proportional to the doses administered [1]. Pharmacokinetics, efficacy, and safety are similar in patients with CKD and those with normal renal function. Hemodialysis does not impact on clearance [2]. The efficacy of etanercept in patients under dialysis for psoriasis [3], RA, and ankylosing spondylitis has been reported in several cases. In our study, hemodialysis did not increase the elimination of etanercept, and treatment was effective against psoriasis in the three cases treated with etanercept. Infliximab is an anti-TNF $\alpha$, with a half-life of about 7 to 12 days. Its maximum concentration, proportional to the administered dose, occurs after the second injection [4]. It is detected in the serum eight weeks after the last dose. Infliximab is effective under hemodialysis, and dialysis was reported not to affect plasmatic concentrations in several patients with Crohn's disease [5], RA, sarcoidosis, and psoriatic arthritis. In our study, it was also effective against psoriasis in patients under hemodialysis. Ustekinumab inhibits IL-12 and IL23. Median half-life is approximately three weeks, and concentration increases proportionally to the administered dose [6]. Its efficacy was reported in four cases of psoriasis under hemodialysis [7, 8]. In our study, with the repetition of dialysis sessions, plasmatic concentrations decreased gradually and became undetectable after 10 weeks, while the half-life was about three weeks, with a moderate loss of efficacy, requiring injections every 10 weeks.

The half-life of ustekinumab is longer than that of etanercept and infliximab, therefore the number of dialysis sessions between two injections is more important and the impact on the elimination of the molecule is probably increased. This phenomenon was not observed with etanercept in our study, nor infliximab in the literature. A molecule with a shorter half-life may therefore be more suitable in patients under hemodialysis.

Dialysis also appears to have an impact on psoriasis. It can be beneficial and transient, with recurrence at a few 